\title{
Rasgos de personalidad en padres del Instituto Tadeo Torres
}

\section{Traits of personality in parents of the Tadeo Torres Institute}

\author{
Arteaga-Rodríguez, José Ignacio ${ }^{1 *}$, Reivan-Ortiz, Geovanny Genaro ${ }^{1}$ y Paredes-Tello, Verónica Isabel $^{1}$ \\ ${ }^{1}$ Universidad Católica de Cuenca \\ *josenacho_17@hotmail.com
}

DOI: https://doi.org/10.26871/killcana_salud.v2i1.201

\begin{abstract}
Resumen
Objetivo: identificar y describir rasgos de personalidad en padres de niños institucionalizados del el hogar infantil Tadeo Torres, en donde se ha podido identificar la resistencia y asimilación a los procesos psicoterapéuticos que aquí se imparten. Contexto: Los usuarios involucrados en esta población específica, tienden a mostrar carencias afectivas de convivencia, en el área familiar. Se ha podido observar escasas habilidades parentales, comportamentales, aunadas a ello, su nivel de educación y conocimientos sobre normas y reglas de interacción familiar son bajos. Al poseer escasa información al respecto, puede provocar que los padres de niños institucionalizados no puedan discernir la importancia de cumplir su función dentro del núcleo familiar, esto sumado a los rasgos de personalidad predominantes puede dificultar la interrelación en su entorno familiar. Materiales y métodos: Para esta indagación se ha determinado utilizar una herramienta psicológica como es el cuestionario de personalidad SEAPsI, el mismo que fue aplicado a una muestra especifica de 16 padres de familia que acuden al Hogar Infantil Tadeo Torres. Resultados: Mediante la indagación a 16 padres de familia que acuden al Hogar Infantil Tadeo Torres de la ciudad de Cuenca se pudo asegurar que los rasgos de personalidad que sobresalen es la de tipo anancastica con un $13 \%$. Conclusiones: el tipo de personalidad en los padres pues este, influye de manera directa al momento de desarrollar habilidades parentales y de crianza con sus hijos, específicamente en esta investigación que según los resultados obtenidos muestran que el tipo de personalidad que prevaleces es la anancástica con un $87,5 \%$ dentro de la esfera afectiva, y tiende a mostrar dificultades de convivencia familiar.
\end{abstract}

Palabras clave: Describir, resistencia, carencias, rasgos, predominan, propuesta.

\begin{abstract}
Objective: identifying and describing personality traits in parents of institutionalized children at the "Tadeo Torres" children's home, they show resistance and assimilation of the psychotherapeutic processes which are taught here. Context: The users involved, in this specific population tend to display a lack of affective coexistence within the family. Parental behavior skills have been found to be poor, and their level of education and knowledge of family norms as well as family interaction rules are low. Because of the lack of information on this topic, the parents of these children may not be able to discern the importance of fulfilling their role within the family nucleus, which, when added to the predominant personality traits may make it difficult for them to interact in a family environment. Materials and methods: The SEAPsI psychological tool has been used for this questionnaire, which was applied to a specific sample of 16 parents who regularly go to "Tadeo Torres" Children's Home. Results: Through the investigation of 16 parents who come to the Tadeo Torres Children's Home in the city of Cuenca, it was possible to assure that the personality traits that stand out are the anancastic type with $13 \%$. Conclusions: the type of personality in the parents because this, directly influences when developing parental and parenting skills with their children, specifically in this research that according to the results show that the personality type that prevails is the ananstic with $87,5 \%$ within the affective sphere, and tends to show difficulties of family coexistence.
\end{abstract}

Key words: Describe, resistance, lacks, features, predominate, proposal.

\section{Introducción}

Los rasgos de personalidad se definen como indicadores que funcionan neuro psíquicamente focalizados en una interacción única de la persona, los mismos pueden tener la capacidad de iniciar y enfocar una reacción frente a distintos impulsos en cada individuo. El estudio de los rasgos de personalidad se puede tomar desde un punto de interés de asumir diferentes posturas de adaptación y personificación dentro de una misma persona sin dejar de ser ellos mismos. ${ }^{1-3}$

Al hablar de rasgos de personalidad existen indicadores recurrentes de cada ser humano por ejemplo; la adaptabilidad, el estado emocional, el temperamento o los valores, mediante de los cuales el sujeto logra interactuar de acuerdo a una reacción frente a estímulos determinados.

En el año 1996 Engl $^{4-6}$ aporta sobre la temática describiendo un análisis profundo al respecto, unido a esto 
Raymond Cattel identificó cuatro rasgos característicos de personalidad adaptativos: a) generales (comunes en todas las personas) específicos (innatos de cada sujeto); b) vacuos (que aparecen a simple vista) interiorizados (se pueden observar mediante un estudio específico de la persona); c) incorporados (acoplados de su aprendizaje) ambientales (influenciados de su interacción y entorno); d) incentivadores (impulsan al sujeto a su objetivo), determinantes (visibilizan para lograr el objetivo y meta planteados). ${ }^{7-9}$

Los rasgos de personalidad pueden incorporarse mediante aprendizajes que se adquieren a través de la experimentación directa con el medio ambiente. Se pueden mencionar dos tipos de caracteres o rasgos de manera generalizada: a) los innatos que se observan específicamente en cada persona, b) los generales que resaltan en muchos sujetos pero gradualmente. ${ }^{10-12}$ Unido a esto se mencionan los rasgos que pueden llegar a acoplarse en distintas situaciones independientemente, es decir, cada individuo es poseedor de sus propios rasgos adaptativos y comportamentales. ${ }^{13}$

Los indicadores de los rasgos de personalidad dan pautas para que el sujeto pueda interactuar y expresarse de manera espontánea, es decir: lo que está pensando y sintiendo frente a determinadas situaciones. ${ }^{14}$ Son distintos los rasgos que se presentan en cada individuo, los cuales pueden variar en grado e intensidad. Al visualizar cual es la respuesta más frecuente del sujeto ante situaciones cambiantes en su entorno, estas respuestas pueden orientar un comportamiento predominante y ser expresadas con regularidad. ${ }^{15}$

Un estudio sobre desintegración familiar realizado por la Universidad de San Carlos de Guatemala en el año 2010, dio a conocer que los rasgos de personalidad y la estabilidad emocional, se presentan cambiantes de manera considerable cuando existe una separación familiar, ${ }^{16}$ unido a ello, los resultados mostraron que el $55 \%$ de los padres presentan tipos de personalidades disociativas.

En el estudio de la personalidad se muestra una frecuente tendencia al uso de técnicas de análisis factorial, al momento de sintetizar instrumentos para el análisis de los rasgos dentro de la estructura de la personalidad huma$\mathrm{na}^{17}$ es decir, se presentan dos corrientes distintas a esta temática, por una parte está la propuesta biológica que se sostiene en la fuerza que ejercen los factores biológicos al momento de mostrar rasgos de personalidad; y por otra está la propuesta léxica, la misma que se basa en la terminología que muestra de forma objetiva las características notables de la personalidad. ${ }^{18}$

Según estas dos propuestas la influencia que puede tener la parte biológica y la parte socio comportamental está equilibrada en un $50 \%$, a partir de esto los caracteres determinantes demuestran una incorporación innata a lo largo de su desarrollo biopsicosocial. Ante esto "Leal, y Vidales (1997), proponen el estudio de la personalidad en 3 dimensiones diferentes: a) pautas y hábitos de organización complementados con aptitudes físicas, las mismas que aportan a un mejor desarrollo social del individuo, b) impulsos internos y presión del ambiente, c) organización sistemática de actitudes y tendencias repetitivas para regular la presión del medio ambiente. ${ }^{18,19}$ El presente estudio tuvo como objetivos: Determinar los rasgos de personalidad predominantes en padres maltratadores de niños institucionalizados en el Hogar Infantil Tadeo Torres; reconocer los rasgos de personalidad prevalentes de padres negligentes en la muestra de estudio; describir los porcentajes obtenidos de padres maltratadores y padres negligentes, así como las variables sociodemográficas de la población de estudio.

\section{Metodología}

Para el desarrollo del estudio, se contó con un diseño no experimental transversal descriptivo, con una muestra no probabilística de selección por voluntarios de un $n=16$ en edades comprendidas entre los 16 a 37 años de edad $(\mathrm{M}=1,25 \mathrm{DT}=, 57)$. Los 16 padres de familia que acuden al hogar infantil Tadeo Torres. Los cuales están conformados por 4 personas de sexo masculino y 12 personas de sexo femenino. Instrumento: Se aplicó el Cuestionario de Personalidad SEAPsI (Balarezo, 2010), el mismo que cuenta con propiedades psicométricas adecuadas a la población ecuatoriana, con una consistencia interna por alfa de Cronbach de .75 respectivamente. El instrumento distingue los rasgos de personalidad predominantes. Mediante selección, muestra 10 tipos distintos de personalidades dentro de la esfera afectiva (histriónica y ciclotímica), cognitivas (anancástica y paranoide), comportamentales (inestable y disocial), y déficit relacional (evitativa, dependiente, esquizoide y esquizotipica), los resultados respuesta varían de entre 0 y 10 . Para cumplir el objetivo de estudio, se utilizó el software estadístico SPSS de IBM versión 24.0 mediante el análisis estadístico descriptivo.

\section{Resultados}

Mediante la indagación a 16 padres de familia que acuden al Hogar Infantil Tadeo Torres de la ciudad de Cuenca se pudo asegurar que los rasgos de personalidad que sobresalen es la de tipo anancastica con un $13 \%$. En tanto a los datos sociodemográficos: el 68,8\% de los padres tienen un nivel de escolaridad básica, 43,8\% viven en unión libre, mientras que solo el 18,8\% están casados. De igual manera se pudo evidenciar que no presentan trastorno de personalidad disruptivo o persistente. Finalmente se determinó que el instrumento SEAPsI presenta una desviación estándar de , $577 \%$ en los resultados.

Seguidamente se detallan las observaciones y resultados que muestran cada uno de los objetivos. 


\subsection{Objetivol}

Tabla 1. Rasgos de personalidad de padres Maltratadores.

\begin{tabular}{|l|l|c|c|}
\hline \multicolumn{2}{|c|}{} & \multicolumn{2}{|c|}{ Tipo de padres } \\
\cline { 3 - 4 } \multicolumn{2}{|c|}{} & \multicolumn{2}{|c|}{ Maltratadores } \\
\cline { 3 - 4 } Afectivas & No presenta & 5 & 31 \\
& Histriónico & 0 & 0 \\
& Ciclotímico & 0 & 0 \\
Cognitivas & No presenta & 2 & 13 \\
& Anancastico & 2 & 13 \\
& Paranoide & 1 & 6 \\
Déficit en la relación & No presenta & 5 & 35 \\
& Inestable & 0 & 0 \\
& Disocial & 0 & 0 \\
& No presenta & 3 & 21 \\
& Evitativo & 1 & 6 \\
& Dependiente & 0 & 0 \\
& Esquizoide & 1 & 6 \\
\hline TOTAL: & Esquizotípico & 0 & 0 \\
\hline
\end{tabular}

Fuente: Stadistics Package for the Social Science 21.0

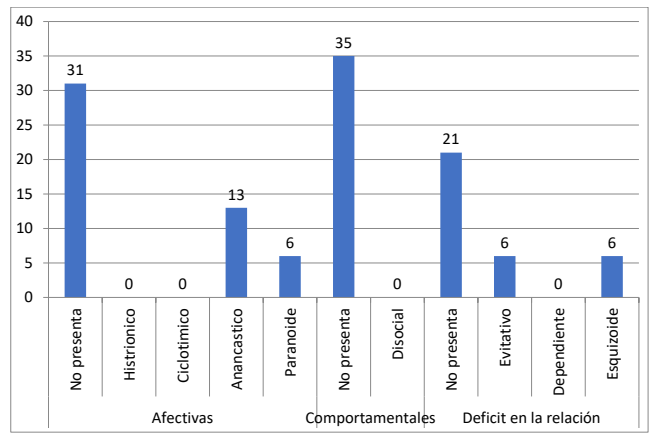

Fig. 1. Frecuencias de personalidad de padres Maltratadores. Fuente: Stadistics Package for the Social Science 21.0

La figura 1 indica que el $13 \%$ de la muestra aplicada posee rasgos de personalidad anancástica, dentro de la esfera cognitiva, el $31 \%$ no presenta personalidades afectivas, el $35 \%$ no presenta personalidades comportamentales y el $21 \%$ no presenta personalidades con déficit relacional.

\subsection{Objetivo 2}

Tabla 2. Rasgos de personalidad de padres Negligentes.

\begin{tabular}{|l|l|c|c|}
\hline \multicolumn{2}{|c|}{} & \multicolumn{2}{|c|}{ Tipo de padres } \\
\cline { 3 - 4 } \multicolumn{2}{|c|}{} & \multicolumn{2}{|c|}{ Negligentes } \\
\cline { 3 - 4 } Cognitivas & No presenta & 9 & 56 \\
& Histriónico & 2 & 13 \\
Compoctivas & Ciclotímico & 0 & 0 \\
& No presenta & 2 & 0 \\
& Anancastico & 6 & 38 \\
Déficit en la relación & Paranoide & 3 & 0 \\
& No presenta & 11 & 6 \\
& Inestable & 0 & 0 \\
& Disocial & 0 & 0 \\
& No presenta & 11 & 0 \\
& Evitativo & 0 & 0 \\
& Dependiente & 0 & 0 \\
\hline TOTAL: & Esquizoide & 0 & 0 \\
& Esquizotípico & 0 & 0 \\
\hline
\end{tabular}

Fuente: Stadistics Package for the Social Science 21.0

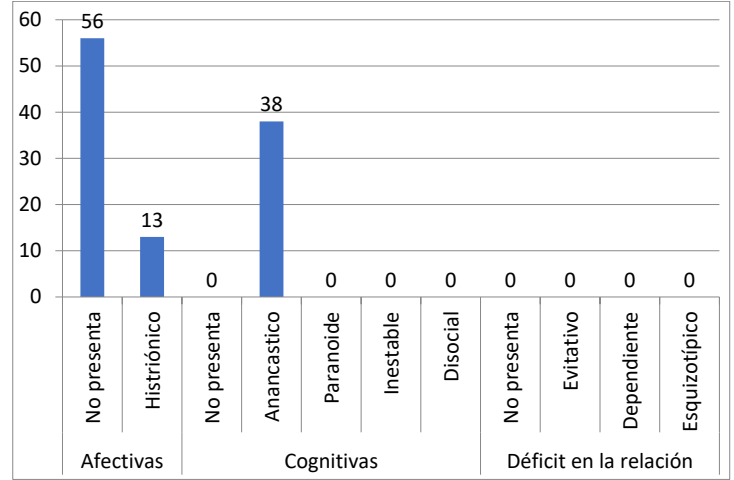

Fig. 2. Porcentaje de personalidad de padres negligentes Fuente: Stadistics Package for the Social Science 21.0

La figura 2 indica que el $38 \%$ de la muestra aplicada posee rasgos de personalidad anancastica, dentro de la esfera cognitiva, el $56 \%$ no presenta personalidades afectivas, el $6 \%$ no presenta personalidades comportamentales y el $0 \%$ no presenta personalidades con déficit relacional.

\subsection{Objetivo 3}

Tabla 3. Rasgos de personalidad de padres Negligentes.

\begin{tabular}{|ll|c|c|c|}
\hline & & \multicolumn{2}{|c|}{ Tipo de padres } & \multirow{2}{*}{ Total } \\
\cline { 3 - 4 } & & Frecuencia & Porcentaje & \\
\hline Afectivas & No presenta & 5 & 9 & 14 \\
Cotal & Histriónico & 0 & 2 & 2 \\
& & 5 & 11 & 16 \\
\hline \multirow{4}{*}{ Total } & No presenta & 2 & 2 & 4 \\
Comportivas & Anancastico & 2 & 6 & 8 \\
Total & Paranoide & 1 & 3 & 4 \\
\hline Déficit en la relación & & 5 & 11 & 16 \\
& No presenta & 5 & 11 & 16 \\
& & 5 & 11 & 16 \\
\hline \multirow{2}{*}{ Total } & Evitativo & 3 & 11 & 14 \\
& Esquizoide & 1 & 0 & 1 \\
& & 5 & 0 & 1 \\
& & 1 & 11 & 16 \\
\hline
\end{tabular}

Fuente: Stadistics Package for the Social Science 21.0

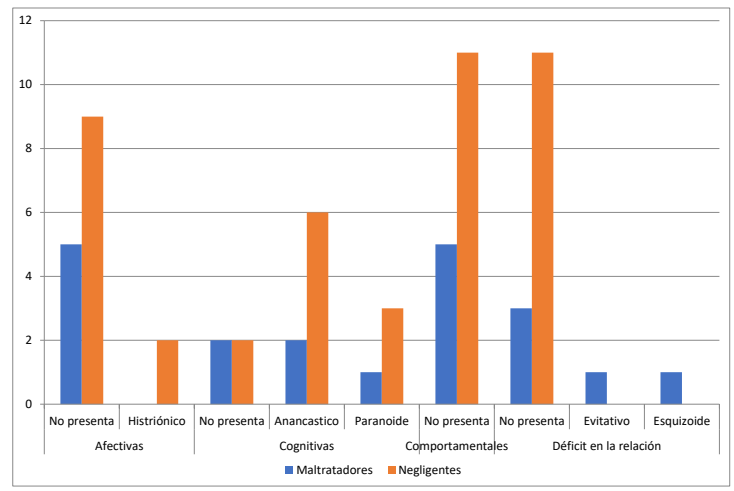

Fig. 3. Porcentaje de personalidades en padres Maltratadores y padres Negligentes.

Fuente: Stadistics Package for the Social Science 21.0 
La figura 3 muestra que el $87,5 \%$ de la muestra no presenta rasgos de personalidad afectivas y el $12,5 \%$ si las presenta, dentro de la esfera cognitiva el $25 \%$ no presenta este rasgo de personalidad, el $50 \%$ presenta rasgos de inclinados a la personalidad anancastica y el $25 \%$ restante presenta inclinación hacia la personalidad paranoide. Dentro de la esfera comportamental el $100 \%$ no presenta este rasgo de personalidades. En cuenta a la esfera de déficit en la relación el $87,5 \%$ no presenta estos rasgos de personalidad en tanto el 6, $25 \%$ presenta rasgos de personalidad evitativas y el $6,25 \%$ presenta rasgos esquizoide.

Tabla 4. Género de padres de familia.

\begin{tabular}{|cl|c|c|}
\hline sexo & Frecuencia & Porcentaje \\
\hline & Femenino & 11 & 68,8 \\
& Masculino & 5 & 31,3 \\
& Total & 16 & 100,0 \\
\hline
\end{tabular}

Fuente: Stadistics Package for the Social Science 21.0

La tabla 4 indica que el $68,8 \%$ de la muestra de padres corresponden al sexo Femenino y el $31,1 \%$ representa el sexo Masculino.

Tabla 5. Edad de población de estudio.

\begin{tabular}{|cl|c|c|}
\hline edad & & Frecuencia & Porcentaje \\
\hline & Adolescente & 1 & 6,3 \\
& Adulto Joven & 10 & 62,5 \\
& Adultos & 5 & 31,3 \\
& Total & 16 & 100,0 \\
\hline
\end{tabular}

Fuente: Stadistics Package for the Social Science 21.0

La tabla 5 muestra que el $6,3 \%$ de la muestra total representa al rango adolescente, el $62,5 \%$ representa al rango de adulto joven y el $31,3 \%$ corresponde al rango de edad adultos.

Tabla 6. Escolaridad.

\begin{tabular}{|cl|c|c|}
\hline escolaridad & & Frecuencia & Porcentaje \\
\hline & Básica & 11 & 68,8 \\
& Bachillerato & 5 & 31,3 \\
& Total & 16 & 100,0 \\
\hline
\end{tabular}

Fuente: Stadistics Package for the Social Science 21.0

La tabla 6 muestra que el 68,8\% de la muestra presenta un nivel de escolaridad básica y el 31,3\% presenta escolaridad bachillerato.

Tabla 7. Estado civil.

\begin{tabular}{|cl|c|c|}
\hline Estado Civil & & Frecuencia & Porcentaje \\
\hline & Soltero & 5 & 31,3 \\
& Casado & 3 & 18,8 \\
& Unión libre & 7 & 43,8 \\
& Divorciado & 1 & 6,3 \\
& Total & 16 & 100,0 \\
\hline
\end{tabular}

Fuente: Stadistics Package for the Social Science 21.0
La tabla 7 muestra que el $31,3 \%$ de padres se encuentran solteros, el $18,8 \%$ están casados, el $43,8 \%$ se encuentran en unión libre y el 6,3\% están divorciados.

Tabla 8. Situación Laboral.

\begin{tabular}{|cl|c|c|}
\hline Laboral & & Frecuencia & Porcentaje \\
\hline & No trabaja & 7 & 43,8 \\
& Trabaja & 9 & 56,3 \\
& Total & 16 & 100,0 \\
\hline
\end{tabular}

Fuente: Stadistics Package for the Social Science 21.0

La tabla 8 muestra que el 43,8 de la muestra total no tiene un trabajo estable y el $56,3 \%$ se encuentra laborando.

\section{Conclusiones y Recomendaciones}

Se concluyó que de los 16 usuarios que acuden al Hogar Infantil Tadeo Torres, el $13 \%$ de los padres maltratadores presentan rasgos de personalidad anancasticos predominantes, así como el $38 \%$ de padres negligentes.

$\mathrm{Al}$ obtener los resultados totales de la muestra y describir los mismos se observó que la tendencia en cuanto a rasgos de personalidad por parte de los 16 padres de familia que acuden al Hogar infantil Tadeo Torres, se observa que el $87,5 \%$ de la muestra no presenta rasgos de personalidad afectivas y el $12,5 \%$ si las presenta, dentro de la esfera cognitiva el $25 \%$ no presenta este rasgo de personalidad, el $50 \%$ presenta personalidades inclinadas a la personalidad anancástica, en tanto $25 \%$ restante presenta inclinaciones Paranoides. Dentro de la esfera comportamental el $100 \%$ no presenta este rasgo de personalidades. En cuenta a la esfera de déficit en la relación el $87,5 \%$ no presenta estos rasgos de personalidad en tanto el $6,25 \%$ presenta rasgos de personalidad evitativas y el $6,25 \%$ presenta rasgos esquizoide.

Con relación a los datos obtenidos, no se han podido observar rasgos de personalidad persistentes en padres de niños institucionalizados, sin embargo, en base a ello, se concluyó que el $6,3 \%$ de la muestra total representa al rango adolescente, el $62,5 \%$ simboliza al rango de adulto joven y el 31,3\% corresponde al rango de edad adultos. En lo referente al género se identificó que el $68,8 \%$ de la muestra de padres corresponden al sexo femenino y el $31,1 \%$ representa el sexo masculino.

La información obtenida puede tener varias pautas de interpretación, según la teoría que subyace al estudio de los rasgos de la personalidad. En el contexto ecuatoriano se evidencia una carente información sobre los análisis de esta mención psicológica, es por esto que se hace propicio indagar más sobre el tema propuesto, para que de esta forma se consoliden nuevos caminos de conocimiento, ya que será mucho mejor el impacto de las intervenciones (primaria, secundaria y terciaria) de los modelos de saludo propuestos por Organización Mundial de la Salud (OMS), para el abordaje de niños maltratados, desplazados, disgregación familiar y violencia intrafamiliar. 
Resulta significativo que el tipo de personalidad en los padres influye de manera directa al momento de desarrollar habilidades parentales y de crianza con sus hijos, específicamente en esta investigación que según los resultados obtenidos muestran que el tipo de personalidad que prevalece es la anancástica con un 87, $5 \%$ dentro de la esfera afectiva, y tiende a mostrar dificultades de convivencia familiar. Estos resultados se contrastan con investigaciones realizadas, ${ }^{20}$ donde se observa que los rasgos de personalidad de padres en un $55 \%$ se inclina por el tipo de personalidades disociativas, las mismas que pueden desembocar en una desintegración total del núcleo familiar. En una investigación realizada en la ciudad de Mexicali se evidencia que la patrones conductuales de los padres tiende a tener personalidades con inclinaciones compulsivas: estos comportamientos influyen en el desarrollo de sus hijos dan- 1 do como resultados, inseguridad, retraimiento, inhibición y agresividad, por ellos se concluye que la personalidad de los padres influyen en el comportamiento de sus hijos provocando en ellos rasgos que pueden ser adaptativos en 11 . su núcleo familiar, los mismos que posteriormente les permitirán crear relaciones sociales según sus aprendizajes. ${ }^{19}$

Para futuras investigaciones se recomienda extender la 13. población de estudio no solo a la localidad dentro de una ciudad sino a toda la urbe ecuatoriana, así mismo, se reco- 14. mienda la utilización de otro instrumento psicológico que mida el diferente constructo teórico para poder describir los posibles sesgos psicométricos del SEAPsI.

\section{Referencias Bibliográficas}

1. Cebrià J, Segura J, Corbella S, Sos $\mathrm{P}$, Comas $\mathrm{O}$, García $\mathrm{M}$, et al. Rasgos de personalidad y burnout en médicos de familia. Atención Primaria. 2001;27(7):459468. Available from: http://linkinghub.elsevier.com/ retrieve/pii/S0212656701788361.

2. R E, L Mn, G JA, S J, V P. Evaluación de la psicopatía infantojuvenil: estudio en una muestra de niños institucionalizados. Anuario de Psicología Jurídica. 2005;15:2340. Available from: http://www.redalyc.org/articulo.oa? $\mathrm{id}=315031849003$.

3. Bonduel Smith AM. Rasgos de personalidad en adolescentes huérfanos diagnosticados con trastornos de la conducta alimentaria. Universidad Rafael Landivar. Guatemala; 2012. Available from: http://biblio3.url.edu.gt/ Tesis/2012/05/42/Bonduel-Aida.pdf.

4. Romero E, Luengo M, Gómez Fraguela JA, Sobral J. La estructura de los rasgos de personalidad en adolescentes: El Modelo de Cinco Factores y los Cinco Alternativos. Psicothema. 2002;14:134-143. Available from: http:// www.redalyc.org/articulo.oa?id=72714119.

5. Burger JM. Personality. 7th ed. Australia ; Belmont, CA: Thomson/Wadsworth; 2008.

6. Gordon A. Capitulo 7 Persona y personalidad. modo del ser humano. Herder. 1985;
7. Cupani M, Pérez ER. Metas de elección de carrera: Contribución de los intereses vocacionales, la autoeficacia y los rasgos de personalidad. Interdisciplinaria. 2006;23(1):81-100. Available from: http://www.scielo. org.ar/scielo.php?script=sci $\left\{\_\right\}$arttext $\{\&\}$ pid=S1668$70272006000100005\{\&\} \operatorname{lng}=\mathrm{es}\{\&\} \mathrm{nrm}=\mathrm{iso}\{\&\}$ tlng=pt.

8. Sinay S. La sociedad de los hijos huérfanos: cuando padres y madres abandonan sus responsabilidades y funciones. Crónica actual. Barcelona: Ediciones B, Grupo Z; 2007.

9. Sánchez Parga J. Orfandades infantiles y adolescentes: introducción a una sociología de la infancia. 1st ed. Quito, Ecuador: Abya Yala : Universidad Politécnica Salesiana : Red Latinoamericana de Maestría en Derechos de Infancia; 2004.

. Schultz DP, Schultz SE. Teorías de la personalidad. Ciencias sociales y humanidades. Thomson; 2002. Available from: https://books.google.com.ec/books?id=6d7sRVMF0cC.

1. Pérez J R . Rasgos de personalidad en hijos de madres solteras.; 2013.

2. Leado, C. Adolescencia y problemas de educación; 2014.

3. Raymond R, Cattell T. Teoría Analítica Factorial de los Rasgos; 2006.

14. Butcher C, Williams J, Graham R, Graham R, Archer $\mathrm{P}$, Tellegen A, et al.. MMPI Inventario Multifásico de Personalidad de Minnesota para Adolescentes; 2012.

15. DiCaprio N. Comportamiento humano. Teorías de la personalidad; 2013.

16. Dávila D. Necesidades psicosociales en los adolescentes; 2014.

17. Jiménez G. Las letras cuentan. Prensa libre; 2012.

18. Chávez. Depresión en adolescentes huérfanos; 2010.

19. Dávila, D. Necesidades psicosociales en los adolescentes.; 2004.

20. Alekseeva LS. Problems of child abuse in the home; 2007.

Recibido: 19 de febrero de 2018

Aceptado: 12 de junio de 2018 
